Bull. Korean Math. Soc. 51 (2014), No. 4, pp. 949-955

http://dx.doi.org/10.4134/BKMS.2014.51.4.949

\title{
A NOTE ON TERNARY CYCLOTOMIC POLYNOMIALS
}

\author{
BIN ZHANG
}

\begin{abstract}
Let $\Phi_{n}(x)=\sum_{k=0}^{\phi(n)} a(n, k) x^{k}$ denote the $n$-th cyclotomic polynomial. In this note, let $p<q<r$ be odd primes, where $q \not \equiv 1$ $(\bmod p)$ and $r \equiv-2(\bmod p q)$, we construct an explicit $k$ such that $a(p q r, k)=-2$.
\end{abstract}

\section{Introduction}

The $n$-th cyclotomic polynomial $\Phi_{n}(x)$ is defined by

$$
\Phi_{n}(x)=\prod_{\substack{1 \leq j \leq n \\(j, n)=1}}\left(x-e^{2 \pi i j / n}\right)=\sum_{k=0}^{\phi(n)} a(n, k) x^{k},
$$

where $\phi$ is the Euler totient function. The coefficients $a(n, k)$ are known to be integral. Let $A(n)$ be the largest absolute value of the coefficients of $\Phi_{n}(x)$. We say that a cyclotomic polynomial is flat if $A(n)=1$. It is easy to see that $A(n)=A(m)$, where $n>1$ is a positive integer and $m$ is the product of the distinct primes dividing $n$. It is also easy to verify that if $n$ is odd, then $A(2 n)=A(n)$. Thus for the purpose of studying coefficients of $\Phi_{n}(x)$, it suffices to consider only odd square-free integers $n$.

Obviously, $\Phi_{p}(x)=\sum_{i=0}^{p-1} x^{i}$ is flat, where $p$ is a prime. Let $\omega(n)$ be the number of distinct odd prime factors of $n$. For square-free $n$, this number $\omega(n)$ is the order of the cyclotomic polynomial $\Phi_{n}(x)$. The case where $\omega(n)=2$ has been studied by several authors (see $[4,8,10,13]$ ), and our understanding of it is rather complete. In particular, the coefficients of $\Phi_{p q}(x)$ are computed in the following lemma. For a proof, see, for example, Lam and Leung [8] or Thangadurai [13].

Received June 25, 2013; Revised November 13, 2013.

2010 Mathematics Subject Classification. 11B83, 11C08, 11N56.

Key words and phrases. cyclotomic polynomial, coefficients of cyclotomic polynomial, ternary cyclotomic polynomial.

This work was supported by Project of Graduate Education Innovation of Jiangsu Province (Grant No. KYLX_0690), the Specialized Research Fund for the Doctoral Program of Higher Education of China (Grant No. 20133207110012) and National Natural Science Foundation of China (Grant No. 10971098). 
Lemma 1.1. Let $p<q$ be odd primes. Let $s$ and $t$ be positive integers such that $p q+1=p s+q$ t written uniquely. Then we have

$a(p q, i)=\left\{\begin{aligned} 1 & \text { if } i=u p+v q \text { for some } 0 \leq u \leq s-1,0 \leq v \leq t-1 ; \\ -1 & \text { if } i=u p+v q-p q \text { for some } s \leq u \leq q-1, t \leq v \leq p-1 ; \\ 0 & \text { otherwise. }\end{aligned}\right.$

There have been extensive studies on the coefficients of cyclotomic polynomials of order three. If $\omega(n)=3$, then $\Phi_{n}(x)$ is also said to be ternary.

Let $3<p<q<r$ be primes satisfying $q \equiv 2(\bmod p)$ and $2 r \equiv-1$ $(\bmod p q)$. In 1936, Lehmer $[9]$ proved that $a(p q r,(p-3)(q r+1) / 2)=(p-1) / 2$, and in 1971, Möller [11] showed that $a(p q r,(p-1)(q r+1) / 2)=(p+1) / 2$.

In 2006, Bachman [1] first established the existence of an infinite family of flat ternary cyclotomic polynomials.

Given odd primes $p<q$, in 2007, Kaplan [7] proved that $A(p q r)=1$ for every prime $r \equiv \pm 1(\bmod p q)$. The author also showed that

$$
A(p q r)=A(p q s)
$$

whenever $s>q$ is a prime congruent to $\pm r(\bmod p q)$.

Let $p<q<r$ be odd primes. In 2010, Zhao and Zhang [14] showed that

$$
A(p q r) \leq \min \{\bar{r}, p q-\bar{r}\},
$$

where $\bar{r}$ is the unique integer such that $0 \leq \bar{r} \leq p q-1$ and $\bar{r} \equiv r(\bmod p q)$ (see Bachman and Moree [2] or Elder [5] for different proofs).

In 2012, Elder [5] analyzed the coefficients of $\Phi_{n}(x)$ by considering it as a gcd of simpler polynomials. In the case where $r \equiv \pm 2(\bmod p q)$, the author used this theory to prove that $A(p q r)=1$ if and only if $q \equiv 1(\bmod p)$.

There are also papers on the coefficients of inverse cyclotomic polynomials (see Moree [12], Bzdȩga [3]) and on maximum gap in (inverse) cyclotomic polynomials (see Hong, Lee, Lee and Park [6]).

In this note, we continue the discussion of ternary cyclotomic polynomials. Our purpose here is to establish the following main result, giving a prescribed coefficient of ternary cyclotomic polynomial $\Phi_{p q r}(x)$ which equals -2 .

Theorem 1.2. Let $p<q<r$ be odd primes, where $q=k p+\ell$ for some $2 \leq \ell \leq p-1$, and $r \equiv-2(\bmod p q)$.

(a) If $\ell$ is odd, then a $(p q r, p q r-p r-2 q r+p-\ell-2)=-2$.

(b) If $\ell$ is even, then a $(p q r, p q r-p r-2 q r+\ell r+\ell-2)=-2$.

Together with (1.1), (1.2) and Theorem 1.2, we obtain:

Corollary 1.3. Let $p<q<r$ be odd primes such that $r \equiv \pm 2(\bmod p q)$. If $q \not \equiv 1(\bmod p)$, then $A(p q r)=2$.

\section{Preliminaries}

We will first introduce some lemmas which are useful to prove our theorem.

Lemma 2.1. The nonzero coefficients of $\Phi_{p q}(x)$ alternate between +1 and -1 . 
Proof. See Lam and Leung [8].

Let $p<q<r$ be odd primes and $\Phi_{p q r}(x)=\sum_{n=0}^{\phi(p q r)} a(p q r, n) x^{n}$. Kaplan [7] proved the following two lemmas.

Lemma 2.2. Let $p<q<r$ be odd primes. Let $n$ be a non-negative integer and $f(i)$ be the unique value $0 \leq f(i)<p q$ such that

$$
r f(i)+i \equiv n \quad(\bmod p q)
$$

Then

$$
\sum_{i=0}^{p-1} a(p q, f(i))=\sum_{j=0}^{p-1} a(p q, f(q+j)) .
$$

Lemma 2.3. Let $p<q<r$ be odd primes. Let $0 \leq n \leq \phi(p q r)$ be an integer. Put

$$
a^{*}(p q, i)=\left\{\begin{array}{cl}
a(p q, i) & \text { if } r i \leq n \\
0 & \text { otherwise }
\end{array}\right.
$$

We have

$$
a(p q r, n)=\sum_{i=0}^{p-1} a^{*}(p q, f(i))-\sum_{j=0}^{p-1} a^{*}(p q, f(q+j)),
$$

where $f(i)$ is the unique value $0 \leq f(i)<p q$ such that $r f(i)+i \equiv n(\bmod p q)$.

We now provide bounds for the values $s$ and $t$ in the equation $p q+1=p s+q t$ used in the proof of main theorem.

Lemma 2.4. Let $p<q$ be odd primes with $q=k p+\ell$ for some $2 \leq \ell \leq p-1$. Let $s$, $t$ be the unique integers $1 \leq s \leq q-1,1 \leq t \leq p-1$, such that $p q+1=p s+q t$. Then (i) $2 \leq t \leq p-1$; (ii) $s \leq q-k-2$; (iii) $s \geq k+1$.

Proof. (i) Since $t=1$ if and only if $q \equiv 1(\bmod p)$, we have $2 \leq t \leq p-1$.

(ii) To prove this statement, we will show that $p s \leq p(q-k-2)$. Note that $\ell t \equiv 1(\bmod p)$ and $t \geq 2$. So $\ell t \geq p+1$. Then $t k p+\ell t-1 \geq k p+2 p$. Since

$$
\begin{aligned}
p s=p q+1-q t & =p q-(t k p+\ell t-1), \\
p(q-k-2)=p q-k p-2 p & =p q-(k p+2 p),
\end{aligned}
$$

we have $p s \leq p(q-k-2)$, implying that $s \leq q-k-2$, as desired.

(iii) Note that $t=p-1$ if and only if $\ell=p-1$. If $t=p-1$, then

$$
p s=p q+1-q t=q+1=k p+p,
$$

implying that $s=k+1$. If $t<p-1$, then $p s=p q+1-q t \geq 2 q+1>(k+1) p$. So $s \geq k+1$. This completes the proof of Lemma 2.4 . 


\section{The Proof of Theorem 1.2(a)}

For any positive integer $n$, by using the condition of Lemma 2.2, we have

$$
r f(i) \equiv n-i \quad(\bmod p q), \quad 0 \leq f(i) \leq p q-1 .
$$

It follows from $r \equiv-2(\bmod p q)$ that

$$
\begin{aligned}
& f(i+1) \equiv f(i)+\frac{p q+1}{2} \quad(\bmod p q) ; \\
& f(i+2) \equiv f(i)+1 \quad(\bmod p q) .
\end{aligned}
$$

In this section, let $q=k p+\ell, 3 \leq \ell \leq p-2$ and $\ell$ is odd. We will show that $a(p q r, n)=-2$, where

$$
n=p q r-p r-2 q r+p-\ell-2 .
$$

Since $3 \leq \ell \leq p-2$, we have $p \geq 5$ and $t \leq p-2$. For $p q+1=p s+q t$, where $s$ and $t$ are positive integers, by Lemma 2.4, we have

$$
2 \leq t \leq p-2 \text { and } s \leq q-k-2 .
$$

In order to use Lemma 2.3, we need to determine for which $k$ will $r f(k)>n$. We will now prove that $r f(k)>n$ whenever $k \in\{p-\ell, p-\ell+2, \ldots, p-1\} \cup$ $\{q+1, q+3, \ldots, q+p-2\}$, and $r f(k) \leq n$ whenever $k \in\{0,2, \ldots, p-\ell-2\} \cup$ $\{1,3, \ldots, p-2\} \cup\{q, q+2, \ldots, q+p-1\}$.

It follows from $(2.1),(3.1)$ and $(3.2)$ that $f(p-\ell)=p q-p-2 q+1, f(p-$ $\ell-2)=p q-p-2 q ; f(p-2)=\frac{p q+\ell}{2}-p-2 q ; f(q+p-1)=\frac{p q-3 q}{2}-p+\frac{\ell+1}{2}$; $f(q+p-2)=p q-p-\frac{3 q-\ell}{2}, f(q+1)=p q-\frac{3 p+3 q}{2}+\frac{\ell+3}{2}$. Then one readily verifies the assertion.

Together with Lemma 2.3, we obtain that

$$
\begin{aligned}
a(p q r, n) & =\sum_{i=0}^{p-1} a^{*}(p q, f(i))-\sum_{j=0}^{p-1} a^{*}(p q, f(q+j)) \\
& =\sum_{i=0}^{\frac{p-3}{2}} a(p q, f(2 i+1))+\sum_{i=0}^{\frac{p-\ell}{2}-1} a(p q, f(2 i))-\sum_{j=0}^{\frac{p-1}{2}} a(p q, f(q+2 j)) .
\end{aligned}
$$

Applying Lemma 2.2 to the above equation yields

$$
\begin{aligned}
a(p q r, n)= & \sum_{j=0}^{\frac{p-3}{2}} a(p q, f(q+2 j+1))-\sum_{i=0}^{\frac{p-1}{2}} a(p q, f(2 i))+\sum_{i=0}^{\frac{p-\ell}{2}-1} a(p q, f(2 i)) \\
& =\sum_{j=0}^{\frac{p-3}{2}} a(p q, f(q+2 j+1))-\sum_{i=\frac{p-\ell}{2}}^{\frac{p-1}{2}} a(p q, f(2 i)) .
\end{aligned}
$$

It is easy to see

$$
f(p-\ell)=(s-1) p+(t-2) q \text { and } 0 \leq t-2<t-1 ;
$$




$$
\begin{aligned}
& f(q+p-2)=\left(q-\frac{k}{2}-1\right) p+(p-1) q-p q \text { and } \\
& s<q-\frac{k}{2}-1<q-1 .
\end{aligned}
$$

Thus, by Lemma 1.1, we have

$$
a(p q, f(p-\ell))=1, \quad a(p q, f(q+p-2))=-1 .
$$

So equation (3.3) becomes

$$
a(p q r, n)=\sum_{j=0}^{\frac{p-5}{2}} a(p q, f(q+2 j+1))-1-\sum_{i=\frac{p-\ell}{2}+1}^{\frac{p-1}{2}} a(p q, f(2 i))-1 .
$$

On invoking Lemma 2.1 we have

$$
\begin{aligned}
\min \{x \mid x>f(p-\ell), a(p q, x) \neq 0\} & =p q-p-2 q+\ell>f(p-1) \\
\max \{y \mid y<f(q+p-2), a(p q, y) \neq 0\} & =p q-\frac{k p}{2}-2 p-q+1<f(q+1) .
\end{aligned}
$$

By using (3.2), we have $f(p-\ell), f(p-\ell+2), \ldots, f(p-1)$ are consecutive integers. So are $f(q+1), f(q+3), \ldots, f(q+p-2)$. Thus we obtain $a(p q, f(2 i))=0$ for $\frac{p-\ell}{2}+1 \leq i \leq \frac{p-1}{2}$ and $a(p q, f(q+2 j+1))=0$ for $0 \leq j \leq \frac{p-5}{2}$.

Therefore, we get

$$
a(p q r, n)=-1-1=-2
$$

as desired.

\section{The Proof of Theorem 1.2(b)}

In this section, let $2 \leq \ell \leq p-1$ and $\ell$ is even, and we will show that $a(p q r, n)=-2$, where

$$
n=p q r-p r-2 q r+\ell r+\ell-2 .
$$

If $p=3, q \equiv 2(\bmod 3)$, we will prove $a(3 q r, q r-r)=-2$. By using $(2.1)$, we obtain $f(0)=q-1, f(2)=q, f(1)=\frac{5 q-1}{2}, f(q)=3 q-1, f(q+2)=0$ and $f(q+1)=\frac{3 q-1}{2}$.

It is clear that

$$
r f(1)>r f(2)>n=r f(0) ; \quad r f(q)>r f(q+1)>n>r f(q+2) .
$$

By using Lemma 2.3, we have

$$
a(3 q r, n)=a(3 q, f(0))-a(3 q, f(q+2)) .
$$

Obviously, $a(3 q, f(q+2))=a(3 q, 0)=1$. We can rewrite $f(0)=q-1=\left(\frac{2 q-1}{3}\right)$. $3+2 \cdot q-3 q$, and so by Lemma 1.1, $a(3 q, f(0))=-1$. Hence, $a(3 q r, q r-r)=-2$.

In what follows, we consider the case $p \geq 5$.

Proceeding as Section 3, for $p q+1=p s+q t, q=k p+\ell$, by Lemma 2.4, we have

$$
2 \leq t \leq p-1 \text { and } k+1 \leq s \leq q-k-2 .
$$


In order to use Lemma 2.3, we need to determine for which $k$ will $r f(k)>n$. We will now prove that $r f(k)>n$ whenever $k \in\{\ell, \ell+2, \ldots, p-1\} \cup\{q+1$, $q+3, \ldots, q+p-2\}$, and $r f(k) \leq n$ whenever $k \in\{0,2, \ldots, \ell-2\} \cup\{1,3, \ldots$, $p-2\} \cup\{q, q+2, \ldots, q+p-1\}$.

It follows from $(2.1),(3.1)$ and $(3.2)$ that $f(\ell)=p q-p-2 q+\ell+1, f(\ell-$ $2)=p q-p-2 q+\ell ; f(p-2)=\frac{p q-p-4 q+\ell}{2} ; f(q+p-1)=\frac{p q-p-3 q+\ell+1}{2}$; $f(q+p-2)=p q-\frac{p+3 q}{2}+\frac{\ell}{2}, f(q+1)=p q-p-\frac{3 q-3}{2}+\frac{\ell}{2}$. Then one readily verifies the assertion.

Note that

$f(\ell)=(s-k-1) p+(t-1) q$ and $0 \leq s-k-1<s-1$;

$f(q+p-2)=\left(q-1-\frac{k-1}{2}\right) p+(p-1) q-p q$ and $s \leq q-1-\frac{k-1}{2} \leq q-1$.

By Lemma 1.1, we have

$$
a(p q, f(\ell))=1, \quad a(p q, f(q+p-2))=-1 .
$$

Together with Lemma 2.3, Lemma 2.2 and (4.1), we obtain that

$$
\begin{aligned}
a(p q r, n)= & \sum_{i=0}^{p-1} a^{*}(p q, f(i))-\sum_{j=0}^{p-1} a^{*}(p q, f(q+j)) \\
= & \sum_{i=0}^{\frac{p-3}{2}} a(p q, f(2 i+1))+\sum_{i=0}^{\frac{\ell}{2}-1} a(p q, f(2 i))-\sum_{j=0}^{\frac{p-1}{2}} a(p q, f(q+2 j)) \\
= & \sum_{j=0}^{\frac{p-3}{2}} a(p q, f(q+2 j+1))-\sum_{i=0}^{\frac{p-1}{2}} a(p q, f(2 i))+\sum_{i=0}^{\frac{\ell}{2}-1} a(p q, f(2 i)) \\
= & \sum_{j=0}^{\frac{p-3}{2}} a(p q, f(q+2 j+1))-\sum_{i=\frac{\ell}{2}}^{\frac{p-1}{2}} a(p q, f(2 i)) \\
= & \sum_{j=0}^{\frac{p-5}{2}} a(p q, f(q+2 j+1))-1-\sum_{i=\frac{\ell}{2}+1}^{\frac{p-1}{2}} a(p q, f(2 i))-1 .
\end{aligned}
$$

On invoking Lemma 2.1 we have

$$
\min \{x \mid x>f(\ell), a(p q, x) \neq 0\}=p q-2 q+\ell>f(p-1) ;
$$

$\max \{y \mid y<f(q+p-2), a(p q, y) \neq 0\}=p q-\frac{3 p+3 q-\ell}{2}+1<f(q+1)$.

By using (3.2), we have $f(\ell), f(\ell+2), \ldots, f(p-1)$ are consecutive integers. So are $f(q+1), f(q+3), \ldots, f(q+p-2)$. Thus we obtain $a(p q, f(2 i))=0$ for $\frac{\ell}{2}+1 \leq i \leq \frac{p-1}{2}$ and $a(p q, f(q+2 j+1))=0$ for $0 \leq j \leq \frac{p-5}{2}$.

Finally, we have

$$
a(p q r, n)=-1-1=-2 .
$$


This completes the proof of Theorem 1.2.

Acknowledgements. I would like to thank Professor Chun-Gang Ji for useful discussions. I would also like to thank the referee for very valuable comments and helpful suggestions.

\section{References}

[1] G. Bachman, Flat cyclotomic polynomials of order three, Bull. London Math. Soc. 38 (2006), no. 1, 53-60.

[2] G. Bachman and P. Moree, On a class of ternary inclusion-exclusion polynomials, Integers 11 (2011), A8, 14 pp.

[3] B. Bzdȩga, On the height of cyclotomic polynomials, Acta Arith. 152 (2012), no. 4, 349-359.

[4] L. Carlitz, The number of terms in the cyclotomic polynomial $F_{p q}(x)$, Amer. Math. Monthly 73 (1966), 979-981.

[5] S. Elder, Flat Cyclotomic Polynomials: A New Approach, arXiv:1207.5811v1, 2012.

[6] H. Hong, E. Lee, H. S. Lee, and C. M. Park, Maximum gap in (inverse) cyclotomic polynomial, J. Number Theory 132 (2012), no. 10, 2297-2315.

[7] N. Kaplan, Flat cyclotomic polynomials of order three, J. Number Theory 127 (2007), no. $1,118-126$.

[8] T. Y. Lam and K. H. Leung, On the cyclotomic polynomial $\Phi_{p q}(X)$, Amer. Math. Monthly 103 (1996), no. 7, 562-564.

[9] E. Lehmer, On the magnitude of the coefficients of the cyclotomic polynomials, Bull. Amer. Math. Soc. 42 (1936), no. 6, 389-392.

[10] H. W. Lenstra, Vanishing sums of roots of unity, in: Proceedings, Bicentennial Congress Wiskundig Genootschap (Vrije Univ., Amsterdam, 1978), Part II, pp. 249-268, Math. Centre Tracts, 101, Math. Centrum, Amsterdam, 1979.

[11] H. Möller, Über die Koeffizienten des n-ten Kreisteilungspolynoms, Math. Z. 119 (1971), $33-40$.

[12] P. Moree, Inverse cyclotomic polynomials, J. Number Theory 129 (2009), no. 3, 667680 .

[13] R. Thangadurai, On the coefficients of cyclotomic polynomials, in: Cyclotomic fields and related topics (Pune, 1999), 311-322, Bhaskaracharya Pratishthana, Pune, 2000.

[14] J. Zhao and X. K. Zhang, Coefficients of ternary cyclotomic polynomials, J. Number Theory 130 (2010), no. 10, 2223-2237.

School of Mathematical Sciences

NANJING NORMAL UNIVERSITY

NANJing 210023, P. R. CHINA

E-mail address: zhangbin100902025@163.com 